PROCEEDINGS OF THE

AMERICAN MATHEMATICAL SOCIETY

Volume 126, Number 12, December 1998, Pages 3495-3499

S $0002-9939(98) 04471-2$

\title{
CENTRALIZERS IN RESIDUALLY FINITE TORSION GROUPS
}

\author{
ANER SHALEV \\ (Communicated by Lance W. Small) \\ In memory of Brian Hartley
}

\begin{abstract}
Let $G$ be a residually finite torsion group. We show that, if $G$ has a finite 2-subgroup whose centralizer is finite, then $G$ is locally finite. We also show that, if $G$ has no 2-torsion, and $Q$ is a finite 2-group acting on $G$ in such a way that the centralizer $C_{G}(Q)$ is soluble, or of finite exponent, then $G$ is locally finite.
\end{abstract}

\section{INTRODUCTION}

The study of centralizers in torsion groups and in locally finite groups in particular has been a major research project in the past few decades; see Shunkov [S], Hartley [H1], [H2], [H3, Section 3] and the references therein. The purpose of this note is to apply some powerful Lie-theoretic tools (see Zelmanov [Z2] and Bahturin and Zaicev [BZ]) in the study of centralizers in residually finite torsion groups. Well known constructions by Golod [G], Grigorchuk [Gr], Gupta and Sidki [GS], and others, show that a residually finite torsion group $G$ need not be locally finite. However, the local finiteness of $G$ can be deduced under some extra assumptions on $G$. For example, if we assume further that $G$ is soluble, or of finite exponent, then $G$ is locally finite; note that the fact residually finite groups of finite exponent are locally finite is one of the equivalent formulations of Zelmanov's celebrated solution to the Restricted Burnside Problem.

As shown by our main result, it sometimes suffices to assume that certain subgroups of $G$ are soluble, or of finite exponent, in order to infer the local finiteness of $G$. Indeed we have

Theorem 1.1. Let $G$ be a residually finite torsion group with no 2-torsion, acted on by a finite 2-group $Q$. Suppose the centralizer $C_{G}(Q)$ is soluble, or of finite exponent. Then $G$ is locally finite.

This theorem extends a recent result of Shumyatsky [Sh2], dealing with the case where $|Q|=2$ and $C_{G}(Q)$ is abelian; it also extends Zelmanov's solution to the Restricted Burnside Problem for groups of odd exponent (take $Q=1$ in Theorem 1.1).

The assumption that $G$ has no 2-torsion is not required if the centralizer $C_{G}(Q)$ is finite. Indeed, using Theorem 1.1 we can easily deduce the following.

Received by the editors March 25, 1997 and, in revised form, April 23, 1997.

1991 Mathematics Subject Classification. Primary 20F50, 20E36; Secondary 20F40, 17B01.

Supported by the Bi-National Science Foundation United States - Israel, Grant No. 92-00034.

(C) 1998 American Mathematical Society 
Corollary 1.2. Let $G$ be a residually finite torsion group, and suppose $G$ has a finite 2-subgroup $Q$ whose centralizer $C_{G}(Q)$ is finite. Then $G$ is locally finite.

Note that, if $Q$ has order 2, then the conclusion already follows from Shunkov's Theorem [S] (see also Hartley's excellent survey [H1]). The cases $Q=C_{2} \times C_{2}$ and $Q=C_{2^{n}}$ have recently been settled in [Sh1] and [RSh].

For residually nilpotent groups we obtain a bit more. By a recent result of Zelmanov, residually nilpotent torsion groups satisfying an identity are locally finite (this follows from Theorem 1.6 of [Z2]). In the following result we draw a similar conclusion assuming only that the centralizer of a certain automorphism group satisfies an identity.

Proposition 1.3. Let $G$ be a residually nilpotent torsion group with no 2-torsion, acted on by a finite 2-group $Q$. Suppose the centralizer $C_{G}(Q)$ satisfies some nontrivial identity. Then $G$ is locally finite.

Our proof of these results is surprisingly short, and is essentially Lie-theoretic. Lie methods are often used in studying fixed points of group automorphisms (see for instance [HB, Chapter 8]); however, this note seems to be the first application of Zelmanov's work to this subject.

I am grateful to Y. Bahturin and to P. Shumyatsky for sending me their preprints [BZ] and [RSh]; results from these papers play an important role in this note.

\section{Proofs}

In what follows we assume that $G$ is a torsion group with no 2-torsion, and that $Q$ is a finite 2-group acting on $G$. Let $C=C_{G}(Q)$ be the centralizer of $Q$ in $G$. We shall show that, under some suitable assumptions, $G$ is locally finite.

Our starting point is the following lemma by Rocco and Shumyatsky [RSh, Lemma 2.2].

Lemma 2.1. Let $G, Q$ be as above, and let $N \triangleleft G$ be a $Q$-invariant normal subgroup. Then $C_{G / N}(Q)=C_{G}(Q) N / N$.

We note that the assumption that $Q$ is a 2-group is essential to the proof of the lemma.

Now suppose $G$ is residually $p$ for some prime $p \neq 2$, and that the centralizer $C_{G}(Q)$ satisfies some non-trivial group identity. We assume that $G$ is finitely generated, and aim to show it is finite.

Let $D_{n}=D_{n}(G)$ be the $n$th dimension subgroup of $G$ in characteristic $p$. The series $\left\{D_{n}\right\}_{n \geq 1}$, also known as the Zassenhaus-Jennings-Lazard series (see [HB, Chapter 8$]$ ), is a central series with elementary abelian factors. Consider the associated Lie algebra

$$
L(G)=\bigoplus_{n \geq 1} D_{n} / D_{n+1}
$$

defined over the field $\mathbb{F}_{p}$ with $p$ elements. Then $Q$ acts on $L(G)$ by Lie algebra automorphisms. Moreover, Lemma 2.1 shows that the centralizer $C_{L(G)}(Q)$ of $Q$ in the Lie algebra is the subalgebra of $L(G)$ corresponding to $C$, namely

$$
C_{L(G)}(Q)=\bigoplus_{n \geq 1}\left(C \cap D_{n}\right) D_{n+1} / D_{n+1} .
$$

Since $C_{G}(Q)$ satisfies some group identity, the Lie subalgebra $C_{L(G)}(Q)$ is PI (i.e., satisfies some non-trivial Lie identity). 
We record the following recent result of Bahturin and Zaicev [BZ, Theorem 2].

Theorem 2.2. Let $L$ be a Lie algebra over a field $F$, acted on by a finite soluble group $H$ whose order is not divisible by the characteristic of $F$. Suppose the centralizer $C_{L}(H)$ is PI. Then $L$ is PI.

Applying this, we conclude that our Lie algebra $L(G)$ is PI. Next, we use the following result of Zelmanov [Z2, Theorem 1.6].

Theorem 2.3. Let $G$ be a finitely generated periodic residually $p$ group. Suppose the Lie algebra $L(G)$ constructed as above is PI. Then $G$ is finite.

We note that the proof of this result combines a hard theorem of Zelmanov on the nilpotency of some PI Lie algebras [Z2, Theorem 1.7] (see also [Z1, Proposition 2 ] for an important special case) with Lazard's work on $p$-adic analytic groups [L]. The idea is to show first that $L(G)$ is nilpotent, and then to deduce that the pro- $p$ completion of $G$ is $p$-adic analytic, hence linear. This shows that $G$ itself is linear. The conclusion of Theorem 2.3 then follows from the local finiteness of torsion linear groups.

Applying Theorem 2.3 we see that our group $G$ is finite. This proves Proposition 1.3 for residually $p$ groups. Now, if $G$ is residually nilpotent with no 2-torsion, then it decomposes into a direct product of maximal $p$-subgroups $(p \neq 2)$, which are $Q$-invariant and residually $p$. It follows that the maximal $p$-subgroups of $G$ are locally finite, so $G$ is locally finite.

Proposition 1.3 is proved.

In order to prove Theorem 1.1 we need to quote a result of Thompson [T]. We denote the Fitting height (i.e., the nilpotent length) of a soluble group $H$ by $h(H)$. For a positive integer $n$, let $l(n)$ denote the number of primes (counted with multiplicity) appearing in the prime factorization of $n$.

Theorem 2.4. Let $H$ be a finite soluble group acted on by a finite group $K$, and suppose $(|H|,|K|)=1$. Let $l(|K|)=k$. Then $h(H) \leq 5^{k} h\left(C_{H}(K)\right)$.

See also $[\mathrm{K}]$ and $[\mathrm{Tu}]$ for improvements of this bound.

We can now prove Theorem 1.1. Suppose first that the centralizer $C=C_{G}(Q)$ is soluble, and denote its Fitting height by $l$. Let $N \triangleleft G$ be a $Q$-invariant subgroup of finite index, and consider the factor group $G / N$, which is acted on by $Q$. Then $G / N$ has odd order, hence it is soluble [FT]. By Lemma 2.1 we have $C_{G / N}(Q)=C N / N$. It follows that $h\left(C_{G / N}(Q)\right) \leq h(C)=l$. Suppose $Q$ has order $2^{k}$ and set $m=5^{k} l$. Applying Thompson's Theorem we obtain

$$
h(G / N) \leq m .
$$

We conclude that $G$ is residually (finite of Fitting height $\leq m$ ). It follows that $G$ has a series of characteristic subgroups

$$
G=G_{0} \geq G_{1} \geq \ldots \geq G_{m}=1,
$$

such that the factors $G_{i} / G_{i+1}$ are all residually nilpotent.

Clearly, $Q$ acts on each factor $G_{i} / G_{i+1}$ in such a way that the centralizer $C_{G_{i} / G_{i+1}}(Q)$ is soluble (being isomorphic to a section of $C$ ). Applying Proposition 1.3 we conclude that the groups $G_{i} / G_{i+1}$ are all locally finite. Hence $G$ is locally finite.

Note that if we replace the solubility of $C$ by the assumption that $C$ satisfies an identity $w$ with the property that finite soluble groups satisfying $w$ have bounded 
Fitting height, then all our arguments go through. By the following consequence of the Hall-Higman theory, this is the case if $w$ is the identity $x^{n}=1$.

Lemma 2.5. The Fitting height of finite soluble groups of exponent $n$ is bounded above by some function of $n$.

Proof. Write $n=p_{1}^{e_{1}} \cdots p_{k}^{e_{k}}$ where $p_{i}$ are distinct primes, and let $H$ be a finite soluble group of exponent $n$. Then the $p_{i}$-length $l_{p_{i}}(H)$ of $H$ is at most $2 e_{i}$ (see [HB, Chapter 9]), and this yields

$$
h(H)<\prod_{i=1}^{k}\left(l_{p_{i}}(H)+1\right) \leq \prod_{i=1}^{k}\left(2 e_{i}+1\right) .
$$

The result follows.

The proof of Theorem 1.1 is now complete.

Remark. In a similar manner, it suffices to assume in Theorem 1.1 that $C_{G}(Q)$ satisfies an Engel condition, or that $C_{G}(Q)$ has a finite normal series such that each of its factors is either soluble or of finite exponent, in order to deduce the local finiteness of $G$.

More generally, it would be interesting to find out which identities bound the Fitting height of finite soluble groups, and thereby to produce various variations on Theorem 1.1.

Finally, let us prove Corollary 1.2. Let $G$ be a residually finite torsion group, and let $Q \leq G$ be a finite 2 -subgroup whose centralizer $C_{G}(Q)$ is finite. Then $G$ has a finite index $Q$-invariant subgroup $G_{1}$ such that $G_{1} \cap C_{G}(Q)=1$. Hence $Q$ acts on $G_{1}$ and $C_{G_{1}}(Q)=1$. By [RSh, Lemma 2.1], $G_{1}$ has no 2-torsion. Applying Theorem 1.1 (using the solubility of the trivial group!) we conclude that $G_{1}$ is locally finite. Therefore $G$ is locally finite, as required.

\section{REFERENCES}

[BZ] Y.A. Bahturin and M.V. Zaicev, Identities of graded algebras, to appear in J. Algebra.

[FT] W. Feit and J.G. Thompson, Solvability of groups of odd order, Pacific J. Math. 13 (1963), 775-1029. MR 29:3538

[G] E.S. Golod, On nil-algebras and residually finite groups, Izv. Akad. Nauk SSSR, Ser. Mat. 28 (1964), 273-276. MR 28:5082

[Gr] R.I. Grigorchuk, On the Burnside problem for periodic groups, Funct. Anal. Appl. 14 (1980), 53-54. MR 81m:20045

[GS] N. Gupta and S. Sidki, On the Burnside problem for periodic groups, Math. Z. 182 (1983), 385-386. MR 84g:20075

[H1] B. Hartley, Centralizers in locally finite groups, Proc. 1st Bressanone Group Theory Conference 1986, Lecture Notes in Math. 1281, Springer, 1987, pp. 36-51. MR 89c:20058

[H2] B. Hartley, A theorem of Brauer-Fowler type and centralizers in locally finite groups, Pacific J. Math. 152 (1992), 101-117.

[H3] B. Hartley, Simple locally finite groups, Proc. NATO ASI on Finite and Locally Finite Groups - Istanbul 1994, Kluwer, 1995, pp. 1-44. MR 96k:20076

[HB] B. Huppert and N. Blackburn, Finite Groups II, Springer, Berlin, 1982. MR 84i:20001a

[K] H. Kurzweil, $p$-Automophismen von auflösbaren $p^{\prime}$-Gruppen, Math. Z. 120 (1971), 326-354. MR 44:1728

[L] M. Lazard, Groupes analytiques p-adiques, Publ. Math. IHES 26 (1965), 389-603. MR 35: 188

[RSh] N. Rocco and P. Shumyatsky, On periodic groups having almost regular 2-elements, to appear in Proc. Edinburgh Math. Soc. 
[S] V.P. Shunkov, On periodic groups with almost regular involution, Algebra and Logic 11 (1972), 260-272.

[Sh1] P. Shumyatsky, On groups having a four-subgroup with finite centralizers, to appear.

[Sh2] P. Shumyatsky, Nilpotency of some Lie algebras associated with $p$-groups, to appear.

[T] J.G. Thompson, Automorphisms of solvable groups, J. Algebra 1 (1964), 259-267. MR 30:3920

[Tu] A. Turull, Fitting heights of groups and of fixed points, J. Algebra 86 (1984), 555-566. MR 85i:20021

[Z1] E.I. Zelmanov, On periodic compact groups, Israel J. Math. 77 (1992), 83-95. MR 94e:20055

[Z2] E.I. Zelmanov, Lie ring methods in the theory of nilpotent groups, Groups '93, Galway-St Andrews, London Math. Soc. Lecture Note Series 212, 1995, pp. 567-585. MR 96i:20048

Institute of Mathematics, Hebrew University, Jerusalem 91904, Israel 